\title{
Performance Evaluation of the Glunovo® Continuous Blood Glucose Monitoring System in Chinese Participants with Diabetes: A Multicenter, Self- Controlled Trial
}

Ran Meng · Tianwei Gu · Fan Yang · Jie Liu • Qichao Sun •

Dalong Zhu

Received: July 30, 2021 / Accepted: October 7, 2021 / Published online: October 26, 2021

(C) The Author(s) 2021

\section{ABSTRACT}

Introduction: The present study was aimed to evaluate the performance and safety of the Glunovo ${ }^{\circledR}$ real-time continuous glucose monitoring system (CGMS) in monitoring interstitial fluid glucose in adult participants with diabetes (at least 18 years old) using venous blood glucose as control.

Methods: This was a multicenter, self-controlled clinical trial, conducted in participants with diabetes from China, between March 2019 to October 2019. The CGMS was used by all the participants for a 14-day wear-in period. The real-time glucose values measured by Glunovo ${ }^{\circledR}$ CGMS were compared with venous blood glucose values measured by the Entwicklung, Konstruktion und Fertigung (EKF) blood glucose detector. The primary outcomes were the consistency rate of CGMS readings and venous blood glucose values (20/20\% standard).

Results: A total of 78 participants (41 men, 37 women) and 156 CGMS sensors were included

Supplementary Information The online version contains supplementary material available at https:// doi.org/10.1007/s13300-021-01171-2. in the study. Among the included participants, 25 and 53 participants had type 1 and type 2 diabetes, respectively, with median age of 52.50 years (range 32-62 years). The overall agreement rate $(20 / 20 \%)$ was $89.71 \%$ (95\% CI 89.18-90.24\%). It was observed that $99.08 \%$ (95\% CI 98.91-99.24\%) and 99.82\% (95\% CI 99.74-99.89\%) of the measuring points fell within the $\mathrm{A}+\mathrm{B}$ zones of the Clarke error grid analysis and Parkes/consensus error grid analysis, respectively. The mean absolute relative difference was $10.30 \% \pm 4.86 \%$. The probability of a glucose measurement falling within a range, when stratified by venous glucose measurements, ranged from $7.14 \%$ for $19.44-$ $22.22 \mathrm{mmol} / \mathrm{L}$ to $79.21 \%$ for $4.44-6.67 \mathrm{mmol} / \mathrm{L}$. There were $73(41.24 \%)$ and $27(57.45 \%)$ successful CGMS alarms for hypoglycemic and hyperglycemic events, respectively.

Conclusion: From the results, Glunovo ${ }^{\circledR}$ CGMS had excellent accuracy and limited clinical risk compared with venous blood glucose in the range of $2.2-22.2 \mathrm{mmol} / \mathrm{L}$ over 14 days.

Keywords: Glunovo®; $\quad$ CGMS; $\quad$ Diabetes; Venous blood glucose

R. Meng · T. Gu $\cdot$ F. Yang $\cdot$ J. Liu .

Q. Sun · D. Zhu ( $\square)$

Department of Endocrinology, Nanjing University

Medical School Affiliated Nanjing Drum Tower

Hospital, Nanjing 210008, China

e-mail: zhudalong@nju.edu.cn 


\section{Key Summary Points}

Why carry out this study?

Traditional glucose monitoring methods provide snapshots of blood glucose concentration and may not provide insights into all the hyper- and hypoglycemic episodes or glycemic variability. Thus, continuous glucose monitoring systems (CGMS) have emerged to address these issues.

Compared to intermittently scanned CGMS (isCGMS), real-time CGMS (rtCGMS) have been demonstrated to provide better glycemic control and quality of life in individuals with diabetes.

Hence, we evaluated the performance and safety of Glunovo ${ }^{\circledR}$ rtCGMS in monitoring interstitial fluid glucose in adult participants with diabetes.

What was learned from the study?

The Glunovo ${ }^{\circ}$ CGMS showed high accuracy in both monitoring the real-time continuous changes and predicting the trend of changes in blood glucose level.

Along with better accuracy, Glunovo ${ }^{\circledR}$ CGMS has a sensor life of 14 days which is an added benefit compared to other CGMS on the market.

\section{INTRODUCTION}

Blood glucose monitoring is essential in glycemic status assessment, prescription of optimal treatment regimen, timely therapy adjustment, and follow-up of patients' glucose status [1]. Traditional methods, like self-monitoring of blood glucose (SMBG) by intermittent capillary sampling, provide snapshots of blood glucose concentration and may not provide insights into all the hyperglycemic and hypoglycemic episodes or glycemic variability [2]. Besides, most patients avoid performing SMBG frequently because of the associated pain and inconvenience [3]. To access long-term glycemic levels, HbA1c is considered the gold standard, as it gives mean glucose levels of the past 2-3 months while the short-term glycemic control cannot be monitored [1]. Continuous glucose monitoring systems (CGMS) have emerged to address the issues with traditional glucose monitoring methods.

CGMS measures blood glucose levels through sensors inserted subcutaneously and thus helps in 24-h continuous monitoring of interstitial glucose and provides accurate data to patients and physicians about blood glucose variability during the measurement period [4]. CGMS also helps in clinical decision-making including titrating drug dosage in patients with diabetes [2]. Currently, two types of CGMS are available, namely flash glucose monitoring (FGM) or intermittently scanned CGMS (isCGMS) and real-time CGMS (rtCGMS) [3]. Previous studies have shown that rtCGMS devices provide better glycemic control compared to FGM/isCGMS [5-7]. But the introduction of the Abbott Freestyle Libre is CGMS, which is more user-friendly and does not require regular calibration, has revitalized the utility of FGM/isCGMS [8, 9].

Nevertheless, previous studies comparing the rtCGMS Dexcom G5 with the Abbott Freestyle Libre isCGMS revealed better performance for rtCGMS with respect to desirable time-in-range for HbA1c glycemic index [4]. Several studies have shown the benefit of rtCGMS in improving glycemic control, reducing hypoglycemia events, and improving quality of life in individuals with type 1 diabetes (T1D) and type 2 diabetes (T2D) [10-13]. This suggests that although the recent generation isCGMS devices might be user-friendly, rtCGMS devices might provide better clinical utility. Wearing of rtCGMS regularly for at least 6 days per week can maximize the clinical benefits of rtCGMS $[10,14]$. It provides insight into the pattern of blood glucose levels and gives warnings to recognize sudden low or high blood glucose levels and helps in preventing fatal episodes $[15,16]$.

Glunovo ${ }^{\circledR}$ is an rtCGMS consisting of a sensor, transmitter, and a mobile application 
for data analysis. The sensor is designed for subcutaneous installation, preferably in the abdomen or forearm, and has a lifetime of 14 days. The sensor generates an electrical signal which is processed by the transmitter and the blood glucose reading is displayed in the mobile application. As the CGMS measures interstitial blood glucose levels, it is important to calibrate CGMS with capillary blood glucose or venous blood glucose [17]. Since, venous blood glucose levels are considered the gold standard for therapy, performance evaluation of CGMS against venous blood glucose levels provides better outcomes [18]. Hence, the present study was aimed to evaluate the performance and safety of Glunovo ${ }^{\circledR}$ rtCGMS in monitoring interstitial fluid glucose in adult participants with diabetes (at least 18 years old) using venous blood glucose as control.

\section{METHODS}

\section{Study Design and Study Population}

The present study was a multicenter, self-controlled clinical trial, conducted to evaluate the performance and safety of the Glunovo ${ }^{\circledR}$ CGMS in Chinese participants with diabetes. A total of 85 participants were enrolled for the study between March 2019 to October 2019, at Gulou Hospital affiliated to Medical College of Nanjing University, Nanjing First Hospital and Tongji Hospital affiliated to Tongji Medical College of Huazhong University of science and technology, and were admitted to the respective hospital for 14 days.

Participants aged 18-70 years old, participants with clinical diagnosis of T1D or T2D for 3 months or more, and participants who had not taken part in any clinical study for the past 3 months were considered for the study. The exclusion criteria included participants with a history of adhesive tape allergy, diabetic ketoacidosis, pregnancy, psychosis, severe allergies, immunosuppressive disorders, or systemic neurological disease or other diseases.

The device has a 14-day real-time glucose oxidase electrochemical sensor with a soft flexible sensor probe. When the sensor probe invades the subcutaneous tissue, glucose and oxygen in the interstitial fluid permeate into the sensor probe, and an electrochemical reaction occurs to generate an electrical signal. This signal is processed by a transmitter $(7 \mathrm{~mm}$ thickness and 3 years of usage life) that sends data of interstitial glucose levels every $3 \mathrm{~min}$, an applicator to apply the transmitter with a single click, and software to store and share data. The applicator was designed to be simple to use and features a button that positions the sensor in place and retracts the introducer needle when pressed. The electric signal processed by the transmitter is converted to blood glucose reading and transmitted to the mobile application through Bluetooth. The application displays the blood glucose reading in real time, reflects the fluctuation trend of blood glucose and generates the trend curve, and can export the historical data. The analysis software is capable of analyzing the data exported from the app and can perform statistical analysis to provide insights into the titration of antidiabetic drugs. Participants were trained to use the system. All sensors were inserted at the clinic using the automated sensor applicator on the abdomen. Two sensors were inserted in each participant for better performance evaluation. The two sensor values in a single participant were calculated by pairwise mean absolute difference (PARD), and matched with the corresponding venous blood glucose values of that particular patient. In case of malfunctioning of one of the sensors, the value from the other sensor was matched with the venous blood glucose values.

The CGMS was used by all the participants for a 14-day wear-in period. As per the sensor's recommendations, the device was calibrated twice every $24 \mathrm{~h}$ with SMBG measurements. After 14 days of wear-in period, paired continuous blood glucose values and venous blood glucose values were collected for each participant. At least 24 readings were collected within $7 \mathrm{~h}$, at different time periods. The paired continuous blood glucose and venous blood glucose readings were collected by randomization, where a random collection period was generated for each participant and divided into three phases: initial, intermediate, and final. The realtime glucose value measured by Glunovo ${ }^{\circledR}$ 
CGMS was compared with venous blood glucose values taken by the respective hospital's nurses and measured by the EKF blood glucose detector (Biosen-C-Line, EKF diagnostics, Cardiff, UK). The study was conducted in accordance with the Declaration of Helsinki 1964 and its later amendments. The study was approved by the ethical committee of Gulou Hospital Affiliated to Nanjing University School of Medicine (Approval no. 2018-246-01), Nanjing First Hospital (Approval no. QX2019031501), and Huazhong University of Science and Technology Drug Clinical Trial Ethics Committee (Approval no. 24.2019). Before study initiation, all participants provided written informed consent.

\section{Study Outcomes}

The primary outcomes of the study were the consistency rate of CGMS readings and venous blood glucose values $(20 / 20 \%$ standard) which means the percentage of sensor values that fell within $\pm 1.1 \mathrm{mmol} / \mathrm{L}(20 \mathrm{mg} / \mathrm{dL})$ of the reference values for glucose concentrations no greater than $4.4 \mathrm{mmol} / \mathrm{L} \quad(80 \mathrm{mg} / \mathrm{dL})$ or within $\pm 20 \%$ for glucose concentrations greater than $4.4 \mathrm{mmol} / \mathrm{L} \quad(80 \mathrm{mg} / \mathrm{dL}) \quad$ [2]. According to Chinese guidelines for technical review of CGMS, the statistically critical value for agreement at the deviation of $\pm 20 \%$ / $20 \mathrm{mg} / \mathrm{dL}$ was greater than 65\%. Apart from consistency rate, the proportion of points falling in the $A+B$ area in Clarke error grid analysis, Parkes (consensus) error grid analysis, and the mean absolute relative difference (MARD) value were assessed for performance evaluation. For the error grid analyses, greater than $95 \%$ of the $\mathrm{A}+\mathrm{B}$ area is considered as the standard [19]. For the MARD, on the basis of available guidelines, a value of less than $18 \%$ was considered as standard for the present study [2].

The secondary outcomes assessed in this study included consistency analysis, accuracy of hyperglycemia and hypoglycemia alarm rate, stability and repeatability of sensor, sensor life and instrument operation score. For the consistency analysis, the rates of change in CGMS glucose reading $(\mathrm{mg} / \mathrm{dL} / \mathrm{min})$ and $\mathrm{EKF}$ venous glucose $(\mathrm{mg} / \mathrm{dL} / \mathrm{min})$ were stratified according to the different change rate levels and the proportion of measurement points falling in the same change rate range were investigated as demonstrated in Table 1 . When the reading on CGMS glucose reading dropped rapidly (less than $-0.11 \mathrm{mmol} / \mathrm{L} / \mathrm{min}$ ), the probability of venous glucose concentration decreasing at less than $-0.11 \mathrm{mmol} / \mathrm{L} / \mathrm{min}$ is $20.88 \%$. Similarly, when the CGMS glucose reading rapidly increased at greater than $0.11 \mathrm{mmol} / \mathrm{L} / \mathrm{min}$, the probability of venous glucose concentration increasing at greater than $0.11 \mathrm{mmol} / \mathrm{L} / \mathrm{min}$ was $35.18 \%$ (Table 1). The hypoglycemia alarm prompt value was set at less than $3.9 \mathrm{mmol} / \mathrm{L}$ while the hyperglycemia alarm prompt value was set at greater than $16.7 \mathrm{mmol} / \mathrm{L}$. For stability analysis, the samples were collected at three different stages: the initial stage (day 1 or 2), the intermediate stage (day $7 \pm 1$ ), and the final stage (day 14). Repeatability of the sensors was calculated by PARD, which is the difference in values between the two sensors at the same timepoint, divided by the mean value of two sensors at the same timepoint. Further, instrument operation score was evaluated using a 12-item questionnaire to assess the participants' satisfaction with the instruments' operation. The device safety was assessed in terms of adverse events screening to monitor clinically significant abnormalities, vital sign measurements, 12-lead electrocardiogram, and laboratory tests such as hematology and liver and kidney function tests. Sensor insertion sites were assessed to monitor infection, bleeding, pain, and skin allergic reactions occurring from sensor implantation.

\section{Statistical Analysis}

The sample size was calculated by considering a consistency rate $(20 / 20 \%)$ of $65 \%$ with an $\alpha$ level of 0.05 and a dropout rate of $20 \% ; 1200$ measurement points were required for a statistical power of $80 \%$. A total of 20 subjects would provide 1200 measurement points with an estimated 60 measurement points per subject. Similarly, for MARD, a total of 60 participants will provide an $80 \%$ statistical power with the 
Table 1 Consistency in the rate of change between paired CGMS glucose readings and venous blood glucose measurements

\begin{tabular}{|c|c|c|c|c|c|c|c|}
\hline \multirow{2}{*}{$\begin{array}{l}\text { Change rate of } \\
\text { reading } \\
(\mathrm{mmol} / \mathrm{L})\end{array}$} & \multicolumn{6}{|c|}{ Change rate of venous blood glucose measurement $(\mathrm{mmol} / \mathrm{L} / \mathrm{min})$} & \multirow{2}{*}{$\begin{array}{l}\text { CGMS venous blood } \\
\text { glucose matching } \\
\text { number }\end{array}$} \\
\hline & $<-0.11$ & $-0.11--0.06$ & $-0.06-0)$ & $0-0.06$ & $0.06-0.11$ & $>0.11$ & \\
\hline$<-0.11$ & $20.88 \%$ & $31.87 \%$ & $27.47 \%$ & $15.38 \%$ & $3.30 \%$ & $1.10 \%$ & 91 \\
\hline$-0.11--0.06$ & $11.95 \%$ & $47.23 \%$ & $33.57 \%$ & $5.41 \%$ & $1.85 \%$ & - & 703 \\
\hline$-0.06-0$ & $1.53 \%$ & $14.33 \%$ & $61.60 \%$ & $20.92 \%$ & $1.35 \%$ & $0.27 \%$ & 6287 \\
\hline $0-0.06$ & $0.57 \%$ & $2.43 \%$ & $29.59 \%$ & $53.84 \%$ & $11.30 \%$ & $2.27 \%$ & 4231 \\
\hline $0.06-0.11$ & $0.55 \%$ & $1.93 \%$ & $7.60 \%$ & $30.52 \%$ & $37.29 \%$ & $22.10 \%$ & 724 \\
\hline$>0.11$ & - & $3.02 \%$ & $9.55 \%$ & $18.09 \%$ & $34.17 \%$ & $35.18 \%$ & 199 \\
\hline
\end{tabular}

$C G M S$ continuous glucose monitoring system

assumed MARD of $18 \% \pm 5 \%$ at an $\alpha$ level of 0.05 with $20 \%$ dropout. In order to meet the sample size requirement of all the study outcomes and endpoints, a total of 60 participants were required for the study. Intention to treat (ITT) principle was used for the performance evaluation (full analysis set). Safety was assessed in the safety dataset that includes all randomized participants with safety evaluation data. For the main evaluation endpoints, the statistical results were presented as point estimation with corresponding 95\% confidence interval (CI). The measurement range of Glunovo ${ }^{\circledR}$ CGMS is ca. $2.2-22.2 \mathrm{mmol} / \mathrm{L}$; the values beyond this range are not included in the analysis. $P \leq 0.05$ was considered as statistically significant. All statistical calculations were performed using SAS 9.4 software.

\section{RESULTS}

\section{Participant Characteristics}

Of the total 85 participants enrolled for the study from three different study centers, seven participants were excluded after screening. Hence, 78 participants (41 men and 37 women) were included for the study with 156 CGMS sensors (one in each participant's left and right abdomen). Among the 156 sensors, a total of three and six sensors fell off from the left and right abdomen, respectively, and so the data points for the entire duration of the study were not available. Paired data from 147 sensors were available for the entire duration of the study (supplementary Fig. 1). Further, among the sensors that fell off, a minimum of one pair of matched data were available for seven sensors and hence a total of 154 sensors were used for performance evaluation. The data from the 154 sensors with matched venous blood glucose data constituted the full analysis set (FAS). Among the included participants, 25 and 53 participants had T1D and T2D diabetes, respectively, with median age of 52.50 years (range 32-62 years). Baseline characteristics of all included participants are presented in Table 2.

\section{Primary Outcomes}

\section{Agreement Analysis}

A total of 12,688 pairs of CGMS and EKF venous blood glucose values were available for performance evaluation. Out of 12,688 pairs of data 2873 measurements were less than $4.4 \mathrm{mmol} / \mathrm{L}$ and 460 were at least $11.1 \mathrm{mmol} / \mathrm{L}$. The overall agreement rate $(20 / 20 \%)$ was $89.71 \%$ (95\% CI $89.18-90.24 \%)$ at the level of measurement points in the ITT dataset, thus meeting the expected standards. 
Table 2 Patient demographics

\begin{tabular}{ll}
\hline Variable & $\begin{array}{l}\text { Number } \\
(\%)\end{array}$ \\
\hline Total & $78(100.00)$ \\
Gender & \\
Male & $41(52.56)$ \\
Female & $37(47.44)$ \\
Marital status & \\
Married & $65(83.33)$ \\
Unmarried & $12(15.38)$ \\
Other & $1(1.28)$ \\
Classification of diabetes mellitus & \\
Type 1 & $25(32.05)$ \\
Type 2 & $53(67.95)$ \\
Past/present medical history & $78(100.00)$ \\
History of surgery & \\
No & $43(55.13)$ \\
Yes & $35(44.87)$ \\
Clinical significance of electrocardiogram & \\
Normal & $54(69.23)$ \\
The abnormality has no clinical & $16(20.51)$ \\
significance & \\
Abnormality has clinical significance & $8(10.26)$ \\
Not checked & $0(0.00)$ \\
\hline & \\
\hline
\end{tabular}

The agreement rate in participants with venous blood concentration less than $3 \mathrm{mmol} / \mathrm{L}$ was $92.86 \%$. For participants with venous blood concentrations of $3-3.9 \mathrm{mmol} / \mathrm{L}, 3.9-10 \mathrm{mmol} /$ $\mathrm{L}$, and $10-13.9 \mathrm{mmol} / \mathrm{L}$, the agreement rates were $62.26 \%, 90.62 \%$, and $88.74 \%$ respectively.

Out of the 697 measurement points with venous blood glucose level greater than $13.9 \mathrm{mmol} / \mathrm{L}$, the agreement rate was $89.47 \%$ (95\% CI 87.32-91.63). Among 12,688 pairs of sensor values, $60.5 \%$ (95\% CI 59.30-61.00\%), $79.31 \% \quad$ (95\% CI $\quad 78.61-80.02 \%), \quad 97.50 \%$ (95\% CI 97.23-97.77\%), and 99.10\% (95\% CI 98.94-99.27\%) were within $\pm 10 \% / 10, \pm 15 \% /$
$15, \pm 30 \% / 30$, and $\pm 40 \% / 40$ of the reference value of venous blood glucose, respectively (Table 3).

\section{Error Grid Analysis}

Clarke Error Grid Analysis All 12,688 pairs of CGMS and EKF venous blood glucose values were considered for Clarke error grid analysis. A scatter plot was constructed using CGMS values and reference venous values on the vertical and horizontal axis, respectively. It was observed that $99.08 \%$ (95\% CI $98.91-99.24 \%)$ of the measuring points fell within the $\mathrm{A}+\mathrm{B}$ zones of the Clarke error grid analysis (Fig. 1a), thereby meeting clinical accuracy. Of $99.08 \%$ that fell in the $\mathrm{A}+\mathrm{B}$ zone, $89.01 \%$ of the measuring points fell in zone A and $10.07 \%$ in zone B while only $0.04 \%$ fell in zone E. The percentage of measurement points falling in $\mathrm{A}+\mathrm{B}$ zones was $40.25 \%$ for a venous blood glucose measurement of $3-3.9 \mathrm{mmol} / \mathrm{L}$, and $99.93 \%$ for a venous blood glucose measurement of 3.9-10 mmol/L. For venous blood glucose measurements of $10-13.9 \mathrm{mmol} / \mathrm{L}$ and greater than $13.9 \mathrm{mmol} / \mathrm{L}$, the percentage of measuring points falling in $\mathrm{A}+\mathrm{B}$ zones was $99.61 \%$ and $99.87 \%$, respectively.

Consensus Error Grid Analysis A total of $99.82 \%$ (95\% CI $99.74-99.89 \%$ ) of the measuring points fell in the $\mathrm{A}+\mathrm{B}$ zone of the Parkes/consensus error grid analysis (Fig. 1b), of which, $89.09 \%$ fell in zone A and $10.73 \%$ fell in zone B. Only $0.02 \%$ fell in zone $\mathrm{D}$, the clinical risk zone, while none fell in zone $\mathrm{E}$. The percentage of measuring points falling in $\mathrm{A}+\mathrm{B}$ zones was $96.86 \%$ for a venous blood glucose measurement of $3-3.9 \mathrm{mmol} / \mathrm{L}$, and $99.88 \%$ for a venous blood glucose measurement of 3.9-$10 \mathrm{mmol} / \mathrm{L}$. For venous blood glucose measurements of $10-13.9 \mathrm{mmol} / \mathrm{L}$ and greater than $13.9 \mathrm{mmol} / \mathrm{L}$, the percentage of measuring points falling in $\mathrm{A}+\mathrm{B}$ zones was $99.79 \%$ and $99.87 \%$, respectively.

\section{Mean Absolute Relative Difference (MARD)}

The MARD was $10.30 \% \pm 4.86 \%$ while the median was $9.38 \%$ (95\% CI 9.53-11.08\%). The MARD values ranged from $35.04 \%$ for a venous 
Table 3 Agreement between paired sensor-reference values in the range of reference glucose levels

\begin{tabular}{|c|c|c|c|c|c|}
\hline $\begin{array}{l}\text { Agreement } \\
\text { level }\end{array}$ & $\begin{array}{l} \pm 10 / 10 \% \\
(\mathrm{mmol} / \mathrm{L})\end{array}$ & $\begin{array}{l} \pm 15 / 15 \% \\
(\mathrm{mmol} / \mathrm{L})\end{array}$ & $\begin{array}{l} \pm 20 / 20 \% \\
(\mathrm{mmol} / \mathrm{L})\end{array}$ & $\begin{array}{l} \pm 30 / 30 \% \\
(\mathrm{mmol} / \mathrm{L})\end{array}$ & $\begin{array}{l} \pm 40 / 40 \% \\
(\mathrm{mmol} / \mathrm{L})\end{array}$ \\
\hline Total & 60.15 & 79.31 & 89.71 & 97.50 & 99.10 \\
\hline $95 \% \mathrm{CI}$ & $59.30,61.00$ & $78.61,80.02$ & $89.18,90.24$ & $97.23,97.77$ & $98.94,99.27$ \\
\hline$<3 \mathrm{mmol} / \mathrm{L}$ & 26.92 & 34.62 & 42.31 & 46.15 & 50.00 \\
\hline $95 \% \mathrm{CI}$ & $9.87,43.97$ & $16.33,52.90$ & $23.32,61.30$ & $26.99,65.32$ & $30.78,69.22$ \\
\hline $3-3.9 \mathrm{mmol} / \mathrm{L}$ & 42.67 & 52.00 & 66.67 & 77.33 & 86.67 \\
\hline $95 \% \mathrm{CI}$ & $31.47,53.86$ & $40.69,63.31$ & $56.00,77.34$ & $67.86,86.81$ & $78.97,94.36$ \\
\hline $3.9-10$ & 59.60 & 78.72 & 89.08 & 97.62 & 99.31 \\
\hline $95 \% \mathrm{CI}$ & $58.60,60.61$ & $77.89,79.56$ & $88.45,89.72$ & $97.30,97.93$ & $99.15,99.48$ \\
\hline $10-13.9$ & 61.97 & 81.35 & 92.42 & 98.59 & 99.45 \\
\hline $95 \%$ CI & $60.14,63.80$ & $79.89,82.82$ & $91.42,93.41$ & $98.15,99.04$ & $99.17,99.73$ \\
\hline$>13.9$ & 63.45 & 83.74 & 91.65 & 95.83 & 98.13 \\
\hline $95 \%$ CI & $59.87,67.03$ & $81.00,86.48$ & $89.60,93.71$ & $94.34,97.31$ & $97.12,99.14$ \\
\hline
\end{tabular}

A

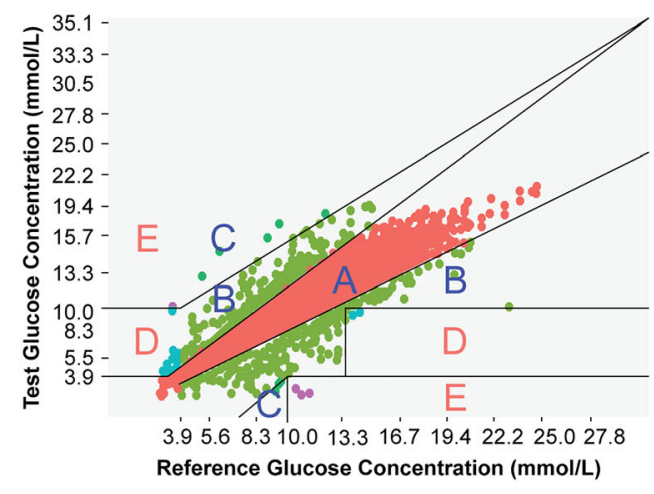

B

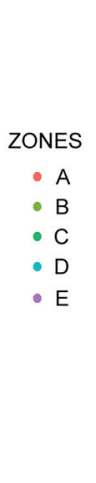

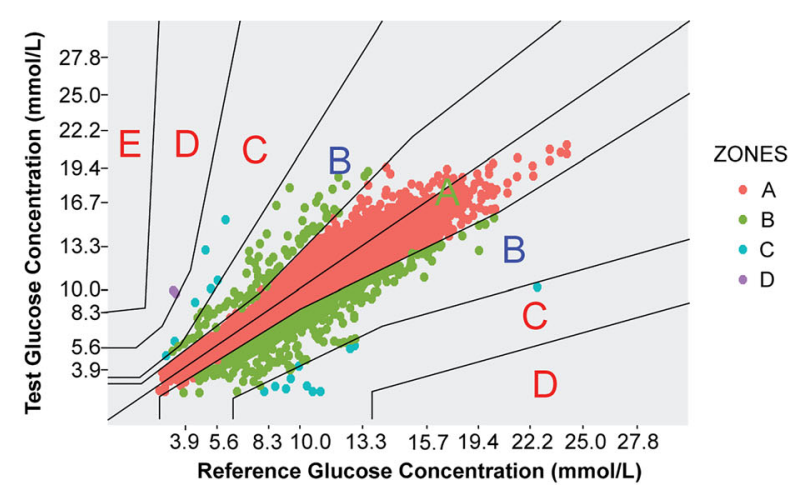

Fig. 1 Error grid analysis of paired sensor-reference values. a Clarke error grid analysis; b Parkes/consensus error grid analysis

blood glucose measurement of less than $3 \mathrm{mmol} / \mathrm{L}$ to $9.91 \%$ for a venous blood glucose measurement of 10-13.9 mmol/L (Table 4).

\section{Secondary Outcomes}

\section{Consistency Analysis}

The probability of a glucose measurement falling within a range, when stratified by venous 
Table 4 Mean absolute relative difference (MARD) value

\begin{tabular}{lllllll}
\hline Index & \multicolumn{6}{l}{ CGMS glucose reading range $(\mathbf{m m o l} / \mathbf{L})$} \\
\cline { 2 - 7 } & $<\mathbf{3 . 0}$ & $\mathbf{3 - 3 . 9}$ & $\mathbf{3 . 9 - 1 0 . 0}$ & $\mathbf{1 0 . 0 - 1 3 . 9}$ & $>\mathbf{1 3 . 9}$ & Total \\
\hline $\mathrm{N}$ (missing) & $6(0)$ & $23(0)$ & $154(0)$ & $136(0)$ & $69(0)$ & $154(0)$ \\
Mean $\pm \mathrm{SD}$ & $35.04 \pm 25.11$ & $19.77 \pm 13.90$ & $10.51 \pm 4.77$ & $9.91 \pm 4.79$ & $10.13 \pm 9.11$ & $10.30 \pm 4.86$ \\
Median $(\mathrm{Q} 1$, & $24.02(15.99$, & $18.43(8.34$, & $9.40(7.47$, & $8.86(6.51$, & $8.25(5.17$, & $9.38(7.46$, \\
Q3) & $61.41)$ & $28.35)$ & $12.45)$ & $12.63)$ & $11.76)$ & $12.08)$ \\
$95 \% \mathrm{CI}$ & $8.68,61.39$ & $13.76,25.78$ & $9.75,11.27$ & $9.10,10.72$ & $7.94,12.31$ & $9.53,11.08$ \\
\hline
\end{tabular}

CGMS continuous glucose monitoring system

Table 5 Hypoglycemia and hyperglycemia alarm detection rate

\begin{tabular}{|c|c|c|c|c|c|c|}
\hline & \multicolumn{3}{|l|}{ Alarm (sensitivity) } & \multicolumn{3}{|c|}{ Detection (specificity) } \\
\hline & Number of alarms & Success, $N(\%)$ & Failure, $N(\%)$ & Number of events & Success, $N(\%)$ & Failure, $N(\%)$ \\
\hline \multicolumn{7}{|c|}{ Hypoglycemia alarm value $(\mathrm{mmol} / \mathrm{L})$} \\
\hline$<3.0$ & 14 & $6(42.86)$ & $8(57.14)$ & 2 & $2(100.00)$ & $0(0.00)$ \\
\hline$<3.3$ & 36 & $13(36.11)$ & $23(63.89)$ & 9 & $7(77.78)$ & $2(22.22)$ \\
\hline$<3.9$ & 177 & $73(41.24)$ & $104(58.76)$ & 69 & $30(43.48)$ & $39(56.52)$ \\
\hline$<4.4$ & 419 & $242(57.76)$ & $177(42.24)$ & 228 & $100(43.86)$ & $128(56.14)$ \\
\hline$<5.0$ & 899 & $678(75.42)$ & $221(24.58)$ & 479 & $196(40.92)$ & $283(59.08)$ \\
\hline$<5.5$ & 1489 & $1260(84.62)$ & $229(15.38)$ & 810 & $295(36.42)$ & $515(63.58)$ \\
\hline \multicolumn{7}{|c|}{ Hyperglycemia alarm detection rate } \\
\hline$>6.7$ & 9357 & $9080(97.04)$ & $277(2.96)$ & 1790 & $739(41.28)$ & $1051(58.72)$ \\
\hline$>7.8$ & 7472 & 7071 (94.63) & $401(5.37)$ & 1726 & $703(40.73)$ & $1023(59.27)$ \\
\hline$>10.0$ & 4083 & $3485(85.35)$ & $598(14.65)$ & 908 & $438(48.24)$ & $470(51.76)$ \\
\hline$>11.1$ & 2889 & $2255(78.05)$ & $634(21.95)$ & 615 & $321(52.20)$ & $294(47.80)$ \\
\hline$>12.2$ & 1801 & $1303(72.35)$ & $498(27.65)$ & 408 & $190(46.57)$ & $218(53.43)$ \\
\hline$>13.3$ & 1043 & 803 (76.99) & $240(23.01)$ & 275 & $136(49.45)$ & $139(50.55)$ \\
\hline$>16.7$ & 243 & $116(47.74)$ & $127(52.26)$ & 47 & $27(57.45)$ & $20(42.55)$ \\
\hline
\end{tabular}

glucose measurements, ranged from $7.14 \%$ for $19.44-22.22 \mathrm{mmol} / \mathrm{L}$ to $79.21 \%$ for $4.44-$ $6.67 \mathrm{mmol} / \mathrm{L}$ (Table 1).

\section{Accuracy of Alarm Rate}

The number of EKF values less than $3.9 \mathrm{mmol} / \mathrm{L}$ was 177 , out of which the number of successful CGMS alarms was 73 (41.24\%). The total 
number of hypoglycemia events was 69 , out of which there were $30(43.48 \%)$ successful events to detect hypoglycemia. The number of EKF values greater than $16.7 \mathrm{mmol} / \mathrm{L}$ was 243; out of these, $116(47.74 \%)$ were successfully alarmed by CGMS. The total number of hyperglycemia events was 47, of which $27(57.45 \%)$ events were successfully alarmed by CGMS (Table 5).

\section{Stability and Repeatability of Sensor}

The results showed no significant difference in the agreement rate $(10 / 10 \%, 15 / 15 \%, 20 / 20 \%$, $30 / 30 \%$, and $40 / 40 \%$ ) and MARD between CGMS and the reference values (Table 6). For repeatability analysis, the mean absolute difference between pairs (PAD) was $0.51 \pm 0.67 \mathrm{mmol} / \mathrm{L}$ (95\% CI $0.50-0.53)$ while the average absolute relative difference between pairs (PARD) was $6.02 \pm 8.08 \% \quad(95 \% \mathrm{CI}$ 5.82-6.22; supplementary Table 1).

\section{Sensor Life and Instrument Operation Score}

A total of 156 sensors were included in the study. Five sensors failed and four were excluded from the study as a result of factors other than device failure. Survival analysis showed that 148 sensors were worn for 14 days with a survival probability of $96.76 \%$ (Fig. 2). The instrument operation score is presented in supplementary Table 2 . None of the participants were dissatisfied with any of the 12 parameters of the CGMS (supplementary Table 2).

\section{Safety Analysis}

Overall, no device-related adverse events were observed in any of the participants. One participant suffered from functional gastrointestinal dysfunction, and had to undergo abdominal CT examination, leading to discontinuation. All the adverse events observed were mild. No remarkable findings were observed from the assessment of vital signs and laboratory findings (Table 7).

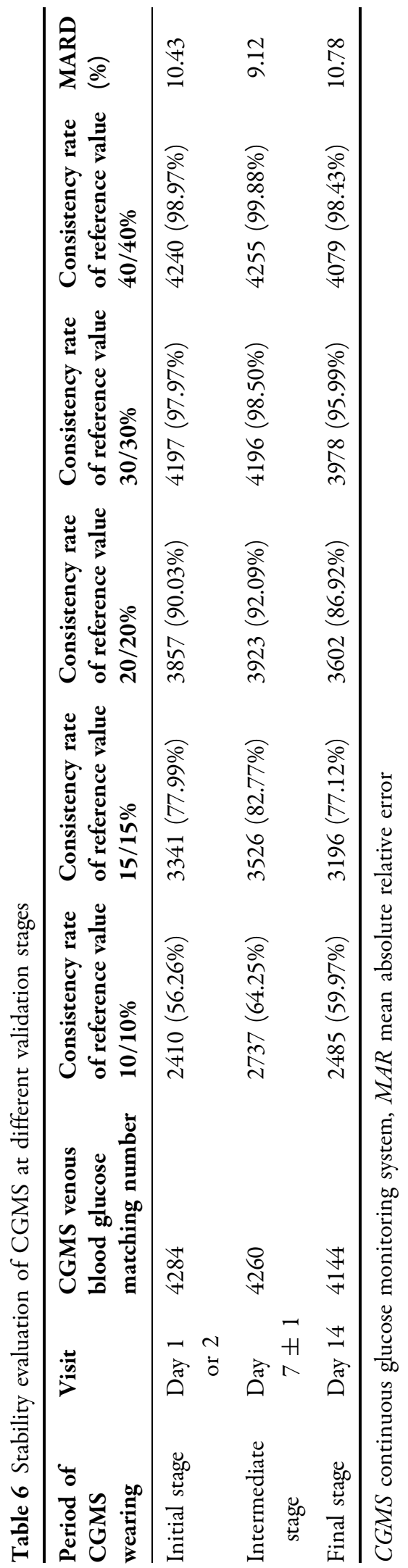




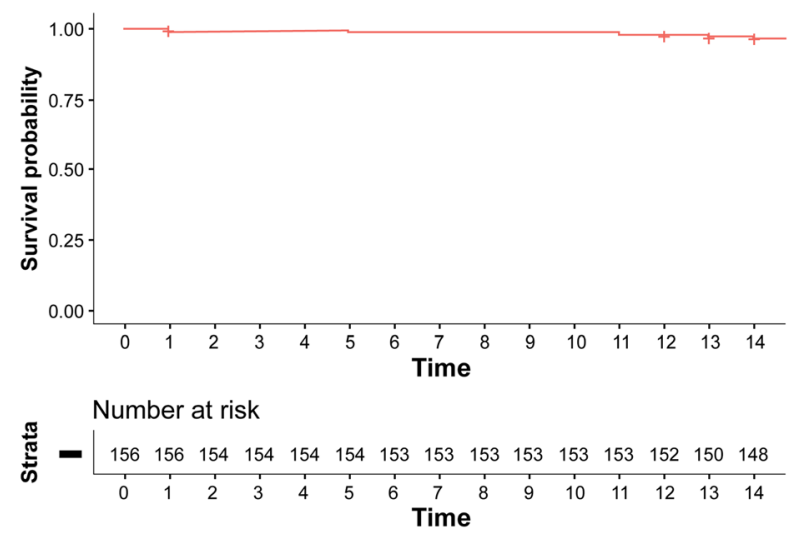

Fig. 2 Kaplan-Meier survival curve of sensor lifetime. The solid red line indicates survival rate; ' + ' represents deletion; shaded region represents $95 \% \mathrm{CI}$ of survival rate

\section{DISCUSSION}

Since the approval of first CGMS by US Food and Drug Administration (FDA) in 1999, the design and performance of rtCGMS have improved markedly [20]. More accurate glucose measurements, increased sensor wear time, reliable alarms, and a user-friendly mobile application are the basic requirements for increased patient acceptance of device and regulatory approval. Glunovo ${ }^{\circledR}$ rtCGMS was developed to increase usability and performance of CGMS; it has a sensor wear time of 14 days and an applicator that reduces the complexity of application. The present study aimed to evaluate the performance and safety of Glunovo ${ }^{\circledR}$ rtCGMS in monitoring interstitial fluid glucose in adult participants with diabetes (at least 18 years old) using venous blood glucose as control. The intricate and voluminous CGMS data were evaluated using numerous statistical methods [21]. Unlike traditional methods that measure plasma glucose levels, CGMS measures interstitial glucose levels; hence it is highly important to assess the accuracy of CGMS $[17,22]$. Further, since CGMS are considered as an alternative for SMBG which are most often performed with capillary blood glucose levels, the performance evaluation of CGMS is frequently assessed by comparing the sensor readings with SMBG. But, better outcomes can be obtained when calibrated using venous blood glucose levels, as venous blood glucose levels are considered the gold standard for designing antidiabetic therapy [18].

In the present study, the accuracy of Glunovo ${ }^{\circledR}$ CGMS was evaluated using agreement rate of CGMS readings and venous blood glucose values $(20 / 20 \%$ standard), the proportion of points falling in the $A+B$ area of error grid analysis, and MARD value. The outcomes analyzed in this study are as per the Chinese guidelines for technical review of CGMS registration which mandates to include agreement rate, error grid analysis, MARD, consistency rate, stability, and repeatability of the sensor. The accuracy of CGMS is evaluated in two aspects, namely numerical accuracy, which

Table 7 Incidence of adverse events

\begin{tabular}{lllc}
\hline & Occurrence & Number of cases & Incidence rate (\%) \\
\hline Adverse event & 98 & 50 & 64.10 \\
Skin reactions & 0 & 0 & 0.00 \\
Serious adverse events & 0 & 0 & 0.00 \\
Serious skin reactions & 0 & 0 & 0.00 \\
Major adverse events & 63 & 33 & 42.31 \\
Adverse events leading to discontinuation & 1 & 1 & 1.28 \\
Skin reactions leading to discontinuation & 0 & 0 & 0.00 \\
\hline
\end{tabular}


includes agreement analysis, and bias analysis and clinical accuracy using error grid analysis.

In the present study, $89.71 \%$ of the sensor readings were within $\pm 20 \%$ of the EKF reading, which was in accordance with specified standards. The agreement rate reported with Dexcom G4 was $81 \%$; this shows the better accuracy of Glunovo ${ }^{\circledR}$ in terms of agreement rate [12]. In comparison to Dexcom G6, the accuracy rates were comparable ( 89.71 vs $92.5 \%$, in adults) [23]. The MARD is another important parameter considered for accuracy analysis of CGMS. The MARD with Glunovo ${ }^{\circledR}$ was $9.38 \%$. The MARD reported with Dexcom G4 and Dexcom G6 was $11 \%$ and $9.8 \%$, respectively $[12,23]$. This indicates better MARD with Glunovo ${ }^{\circledR}$ in adults compared to Dexcom G4 and Dexcom G6, suggesting better performance of Glunovo ${ }^{\circledR}$. From error grid analysis results, $99.08 \%$ and $99.82 \%$ of the sensor measurements fell in the $\mathrm{A}+\mathrm{B}$ zone of Clarke and consensus error grid analysis, respectively. Similar results were reported with Dexcom G4, where $99.5 \%$ of sensor measurements fell in zone A and B of Clarke error grid analysis [12]. For Dexcom G6, 95.7\% of measurements fell in zone A of Clarke error grid analysis [23]. It was reported that $99.1 \%$ and 99.8\% fell in the $\mathrm{A}+\mathrm{B}$ zone of Clarke and consensus error grid analysis, respectively, with Medtrum A6 TouchCare ${ }^{\circledR}$ [2].

The rate of successful hypoglycemia and hyperglycemia detection with Glunovo ${ }^{\circledR}$ was $43.48 \%$ and $57.45 \%$, respectively. In the Guardian Continuous Monitoring System ${ }^{\mathrm{TM}}$, the rate of false hypoglycemia alerts was $47 \%$, and that of hyperglycemia alerts was 19\% [24]. The relatively low accuracy rates of high and low alarms in CGMS are due to the physiological lag of interstitial glucose relative to blood glucose levels, along with device lag [25].

Along with better accuracy, Glunovo ${ }^{\circledR}$ CGMS has a sensor life of 14 days, which is an added benefit compared to other CGMS like Dexcom G4 [12], Dexcom G5 [26], and Medtrum A6 TouchCare ${ }^{\circledR}[2]$ that has 7 days of sensor life, and Dexcom G6 that has 10 days of sensor life [23]. This prolonged life of the sensor helps in patient comfort and hence is an affordable option as it reduces the frequency of changing the sensor. Also, Glunovo ${ }^{\circledR}$ CGMS has a 3-year usage life transmitter with slim design (7 $\mathrm{mm}$ thickness), features Bluetooth low energy wireless technology that sends data to a smart phone every $3 \mathrm{~min}$, and offers a better option compared to the 3 months usage life of Dexcom G6. The device offers a single button smart applicator to apply the transmitter and sensor to the skin with ease. The software allows one to share data with family and healthcare clinicians (up to 10 people). The results of this study are indeed encouraging and offer patients a 14-day sensor wear time, thereby increasing the usability of rtCGMS and thus helping them to have a complete picture of glycemic variations throughout the day.

\section{CONCLUSIONS}

The present study investigated the performance of Glunovo ${ }^{\circledR}$ CGMS in terms of accuracy, stability, repeatability, consistency, and safety. The Glunovo ${ }^{\circledR}$ CGMS showed high accuracy in both monitoring the real-time continuous changes and predicting the trend of changes in blood glucose level. From the results, Glunovo ${ }^{\circledR}$ CGMS had excellent accuracy and limited clinical risk compared with venous blood glucose in the range of $2.2-22.2 \mathrm{mmol} / \mathrm{L}$ over 14 days. This real-time data could be used for clinical decision-making.

\section{ACKNOWLEDGEMENTS}

Funding. No funding or sponsorship was received for this study or publication of this article. The Rapid Service Fee was funded by the authors.

Medical writing, editorial, and other assistance. The authors would like to acknowledge Dr. Satya Lavanya Jakki, Dr. Kaushik Subramanian and Dr. Amit Bhat (Indegene Pvt Ltd) for medical writing and editorial support.

Authorship. All named authors meet the International Committee of Medical Journal Editors (ICMJE) criteria for authorship for this 
article, take responsibility for the integrity of the work as a whole, and have given their approval for this version to be published.

Author Contribution. RM, TG, and DZ contributed to the conception of the study and writing of the manuscript; FY and JL contributed significantly to data acquisition, analysis and interpretation; QS and DZ contributed to critical revisions on the manuscript. All authors read and approved the final manuscript.

Disclosures. Ran Meng, Tianwei Gu, Fan Yang, Jie Liu, Qichao Sun, and Dalong Zhu have nothing to disclose.

Compliance with Ethics Guidelines. The study was conducted in accordance with the Declaration of Helsinki 1964 and its later amendments. The study was approved by the ethical committee of Gulou Hospital Affiliated to Nanjing University School of Medicine (Approval no. 2018-246-01), Nanjing First Hospital (Approval no. QX20190315-01), and Huazhong University of Science and Technology Drug Clinical Trial Ethics Committee (Approval no. 24.2019). Before study initiation, all participants provided written informed consent.

Data Availability. The datasets generated during and/or analyzed during the current study are available from the corresponding author on reasonable request.

Open Access. This article is licensed under a Creative Commons Attribution-NonCommercial 4.0 International License, which permits any non-commercial use, sharing, adaptation, distribution and reproduction in any medium or format, as long as you give appropriate credit to the original author(s) and the source, provide a link to the Creative Commons licence, and indicate if changes were made. The images or other third party material in this article are included in the article's Creative Commons licence, unless indicated otherwise in a credit line to the material. If material is not included in the article's Creative Commons licence and your intended use is not permitted by statutory regulation or exceeds the permitted use, you will need to obtain permission directly from the copyright holder. To view a copy of this licence, visit http://creativecommons.org/licenses/bync/4.0/.

\section{REFERENCES}

1. Bao Y, Chen L, Chen L, et al. Chinese clinical guidelines for continuous glucose monitoring. Diabetes Metab Res Rev. 2019;35:2.

2. Zhou J, Zhang S, Li L, et al. Performance of a new real-time continuous glucose monitoring system: a multicenter pilot study. J Diabetes Investig. 2018;9: 286-93.

3. Bruttomesso D, Laviola L, Avogaro A, et al. The use of real time continuous glucose monitoring or flash glucose monitoring in the management of diabetes: a consensus view of Italian diabetes experts using the Delphi method. Nutr Metab Cardiovasc Dis. 2019;29:421-31.

4. Ajjan RA. How can we realize the clinical benefits of continuous glucose monitoring? Diabetes Technol Ther. 2017;19:27-36.

5. Reddy M, Jugnee N, El Laboudi A, et al. A randomized controlled pilot study of continuous glucose monitoring and flash glucose monitoring in people with type 1 diabetes and impaired awareness of hypoglycaemia. Diabet Med. 2018;35:483-90.

6. Reddy M, Jugnee N, Anantharaja S, et al. Switching from flash glucose monitoring to continuous glucose monitoring on hypoglycemia in adults with type 1 diabetes at high hypoglycemia risk: the extension phase of the I HART CGM study. Diabetes Technol Ther. 2018;20:751-7.

7. Hásková A, Radovnická L, Petruželková L, et al. Real-time CGM is superior to flash glucose monitoring for glucose control in type 1 diabetes: The CORRIDA Randomized Control Trial. 2020. https:// diabetesjournals.figshare.com/articles/figure/Realtime_CGM_is_Superior_to_Flash_Glucose_ Monitoring_for_Glucose_Control_in_Type__1_ diabetes_The_CORRIDA_Randomized_Control_ Trial/12767927. Accessed February 4, 2021.

8. Bolinder J, Antuna R, Geelhoed-Duijvestijn P, et al. Novel glucose-sensing technology and hypoglycaemia in type 1 diabetes: a multicentre, nonmasked, randomised controlled trial. Lancet. 2016;388:2254-63. 
9. Blum A. Freestyle libre glucose monitoring system. Clin Diabetes. 2018;36:203-4.

10. Battelino T, Phillip M, Bratina N, et al. Effect of continuous glucose monitoring on hypoglycemia in type 1 diabetes. Diabetes Care. 2011;34:795-800.

11. Hommel E, Olsen B, Battelino $\mathrm{T}$, et al. Impact of continuous glucose monitoring on quality of life, treatment satisfaction, and use of medical care resources: analyses from the SWITCH study. Acta Diabetol. 2014;51:845-51.

12. Nakamura K, Balo A. The accuracy and efficacy of the Dexcom G4 Platinum continuous glucose monitoring system. J Diabetes Sci Technol. 2015;9: 1021-6.

13. Beck RW, Riddlesworth TD, Ruedy K, et al. Continuous glucose monitoring versus usual care in patients with type 2 diabetes receiving multiple daily insulin injections: a randomized trial. Ann Intern Med. 2017;167:365-74.

14. Garg SK, Voelmle MK, Beatson CR, et al. Use of continuous glucose monitoring in subjects with type 1 diabetes on multiple daily injections versus continuous subcutaneous insulin infusion therapy: a prospective 6-month study. Diabetes Care. 2011;34:574-9.

15. Boland E, Monsod T, Delucia M, et al. Limitations of conventional methods of self-monitoring of blood glucose: lessons learned from 3 days of continuous glucose sensing in pediatric patients with type 1 diabetes. Diabetes Care. 2001;24:1858-62.

16. Kaufman FR, Gibson LC, Halvorson M, et al. A pilot study of the continuous glucose monitoring system: clinical decisions and glycemic control after its use in pediatric type 1 diabetic subjects. Diabetes Care. 2001;24:2030-4.

17. Burge MR, Mitchell S, Sawyer A, et al. Continuous glucose monitoring: the future of diabetes management. Diabetes Spectr. 2008;21:112-9.

18. WHO. Definition and diagnosis of diabetes mellitus and intermediate hyperglycemia: report of a $\mathrm{WHO} /$
IDF consultation. 2006. https://www.who.int/ diabetes/publications/Definition $\% 20$ and $\%$ 20diagnosis\%20of\%20diabetes_new.pdf. Accessed October 5, 2021.

19. Clarke WL, Cox D, Gonder-Frederick LA, et al. Evaluating clinical accuracy of systems for selfmonitoring of blood glucose. Diabetes Care. 1987;10:622-8.

20. Facchinetti A. Continuous glucose monitoring sensors: past, present and future algorithmic challenges. Sensors. 2016;16:2.

21. Clarke W, Kovatchev B. Statistical tools to analyze continuous glucose monitor data. Diabetes Technol Ther. 2009;11(Suppl 1):S45-54.

22. Zhou J, Lv X, Mu Y, et al. The accuracy and efficacy of real-time continuous glucose monitoring sensor in Chinese diabetes patients: a multicenter study. Diabetes Technol Ther. 2012;14:710-8.

23. Shah VN, Laffel LM, Wadwa RP, et al. Performance of a factory-calibrated real-time continuous glucose monitoring system utilizing an automated sensor applicator. Diabetes Technol Ther. 2018;20:428-33.

24. Bode B, Gross K, Rikalo N, et al. Alarms based on real-time sensor glucose values alert patients to hypo- and hyperglycemia: the Guardian Continuous Monitoring System. Diabetes Technol Ther. 2004;6:105-13.

25. McGarraugh G. Alarm characterization for continuous glucose monitors used as adjuncts to selfmonitoring of blood glucose. J Diabetes Sci Technol. 2010;4:41-8.

26. Heinemann L, Freckmann G, Ehrmann D, et al. Real-time continuous glucose monitoring in adults with type 1 diabetes and impaired hypoglycaemia awareness or severe hypoglycaemia treated with multiple daily insulin injections (HypoDE): a multicentre, randomised controlled trial. Lancet. 2018;391:1367-77. 